\title{
ESTRATEGIAS TECNOLÓGICAS CONSERVADAS EN CONTEXTOS AGROPASTORILES TEMPRANOS DE LA PUNA MERIDIONAL ARGENTINA
}

\author{
TECHNOLOGICAL STRATEGIES IN EARLY AGROPASTORAL CONTEXTS \\ FROM THE SOUTHERN PLATEAU OF ARGENTINA
}

\begin{abstract}
Susana Pérez ${ }^{1}$
Las estrategias conservadas y expeditivas son las dos más utilizadas, desde la perspectiva de la organización tecnológica, para explicar el producto material que resulta de su implementación. En general, han sido vinculadas con sociedades de cazadoresrecolectores, por lo que son escasos los intentos de extender su aplicabilidad a sociedades con economías productoras. En este sentido, sobre la base de conjuntos artefactuales de un caso particular de estudio, las palas y/o azadas líticas de Antofagasta de la Sierra (Catamarca, Puna Meridional Argentina), se analiza y discute la implementación de una estrategia tecnológica conservada, vinculada a contextos agropastoriles tempranos y cómo la misma se ve reflejada en algunas variables del diseño de los artefactos. La metodología empleada comprendió tres vías de estudio: análisis tecnomorfológico, experimentación y análisis de rastros de uso.

Palabras claves: sociedades agropastoriles, palas y/o azadas líticas, estrategia conservada, diseño de los instrumentos, enmangue, larga vida útil.
\end{abstract}

From the perspective of technological organization, curated, expedient strategies are the most widely used to explain the resulting material products of their implementation. In general, these strategies have been associated with hunter-gatherer societies, thus their applicability to producer economies has been rare. Based on a particular artifact assemblage of lithic shovels and/or hoes from Antofagasta de la Sierra (Catamarca, Southern Plateau of Argentina), we analyze and discuss the implementation of a technological strategy from an early agropastoral context and how it is reflected in artifact designs. The methodology employed consisted of techno-morphological analysis, experimentation, and use wear analysis.

Key words: Agropastoral societies, lithic shovels and/or hoes, tool designs, preserved strategy, hafting, extended use-life.

La abundancia de palas y/o azadas líticas en la ergología de sitios arqueológicos del noroeste Argentino marca la necesidad de profundizar las investigaciones acerca del rol que jugaron estos instrumentos en la organización de la tecnología lítica de sociedades con economías productoras de alimentos.

El objetivo del estudio de la organización tecnológica es determinar qué estrategias o combinación de estrategias fueron usadas y cómo se reflejan en el comportamiento y cambio cultural (Carr 1994a). Siguiendo a Nelson, se entiende como organización tecnológica "the selection and integration of strategies for making, using, transporting, and discarding tools, and the materials needed for their manufacture and maintenance" (Nelson 1991:57). Dichas estrategias constituyen etapas cruciales para entender la organización y complejización de la tecnología, conformando de este modo la base para establecer la dinámica del comportamiento tecnológico.

En este sentido, a fin de acercarnos a la comprensión de la relación de este tipo de instrumentos con los sistemas económicos de producción de alimentos, se apunta a establecer esa relación desde un punto de vista tecnofuncional y el rango de actividades llevadas a cabo. De este modo, dentro de los objetivos perseguidos se encuentran: estudiar la tecnología involucrada en la manufactura de estos instrumentos, caracterizar los materiales, comprobar los efectos del enmangamiento, identificar el área usada, determinar la forma de utilización y establecer las actividades desarrolladas.

A partir del registro arqueológico, se aborda la discusión acerca de la implementación de una estrategia tecnológica conservada, vinculada a contextos agropastoriles tempranos y cómo la misma se ve reflejada en algunas variables del diseño de los artefactos.

1 Instituto Nacional de Antropología y Pensamiento Latinoamericano y Pontificia Universidad Católica Argentina, Buenos Aires, Argentina.superez@speedy.com.ar 
El presente trabajo intenta realizar un aporte en relación a las estrategias tecnológicas implementadas y constituye un primer acercamiento a aspectos relacionados con la organización tecnológica. Si bien la clasificación de conservado y expeditivo puede resultar en una explicación simplificada, es indudable que la caracterización de los artefactos y su contexto reflejan las diferentes maneras de implementar las estrategias conservadas y expeditivas, y el uso de estos conceptos resulta útil para identificar los tipos de planes y explicar el comportamiento humano (Nelson 1991:62).

Las inferencias fueron realizadas a partir del análisis de los materiales arqueológicos, así como también de datos empíricos generados en el programa experimental. Los resultados provienen de las investigaciones que se vienen realizando desde hace varios años en el Departamento de Antofagasta de la Sierra (Catamarca, Puna Meridional Argentina), correspondientes al análisis de palas y/o azadas líticas recuperadas del sitio Casa Chávez Montículos (Montículos 1 y 4).

\section{El Caso de Estudio}

El sitio CCHM (Montículos 1 y 4) se encuentra ubicado en el Departamento de Antofagasta de la Sierra (Provincia de Catamarca) entre los $25^{\circ} 50^{\prime}$ y $26^{\circ} 10^{\prime}$ de Lat. sur y entre los $67^{\circ} 30^{\prime}$ y $67^{\circ} 10^{\prime}$ de Long. oeste (Figura 1), siendo su valor promedio altitudinal entre los 3.450 y 3.550 msm (Olivera 1991a, 1991b). Antofagasta de la Sierra constituye una de las cuencas hidrográficas endorreicas que posee cursos de aguas permanentes o semipermanentes, lo que origina vegas de buena pastura y ambientes relativamente aptos para el cultivo (Olivera 1991a). De este modo, es posible observar la presencia de pastizales en los valles abrigados, en la ribera de las lagunas, vegas y márgenes de los cursos de agua.

En el territorio mencionado se presentan variaciones microambientales que permiten distinguir, en primera instancia, tres sectores de muestreo: 1) Fondo de Cuenca, 2) Sectores Intermedios $y$ 3) Quebradas de Altura, los cuales se diferencian topográficamente ofreciendo recursos también diferenciales (Olivera 1991a, 1991b). Al mismo tiempo, la muestra de sitios no es homogénea en cuanto a su calidad y tipo de información; además la conservación del registro arqueológico no es la misma.
El sitio CCHM, ubicado en el microsector de muestreo del Fondo de Cuenca, es la unidad arqueológica analizada en este trabajo. Se compone de una serie de 10 estructuras monticulares artificiales de dimensiones variables, dispuestas sobre una superficie de alrededor de $300 \mathrm{~m}$ por $300 \mathrm{~m}$, distribuidos en dos grupos sobre sectores de terreno más elevados y alrededor de un espacio central deprimido (Olivera 1991b). Entre ellos, el Montículo 1 evidencia una ocupación larga y recurrente con, por lo menos, un claro episodio de abandono del sitio (Olivera y Nasti 1993). Hasta el presente, se realizaron excavaciones sistemáticas sólo en los Montículos 1 y 4 . El registro arqueológico lo vincula con ocupaciones agropastoriles tempranas y ha sido interpretado como un sitio Base Residencial de Actividades Múltiples; los análisis radiocarbónicos arrojan fechados comprendidos entre $1.530 \pm 70$ a.p. y $2.120 \pm 60$ a.p. (Olivera y Vigliani 2000-2002: Cuadro 2). Por otro lado, el sitio denominado Bajo del Coypar I-Sector I (Figura 1), ubicado en el microsector de muestreo del Fondo de Cuenca, fue caracterizado por Olivera (1991b; Olivera y Vigliani 2000-2002) como un sitio de producción agrícola, consta de una superficie de 397 ha y se halla en las inmediaciones del sitio CCHM.

\section{Tecnología Conservada y Expeditiva}

A partir del trabajo de Binford (1969, 1973, 1979, 1980), el estudio de la tecnología desde una perspectiva organizativa fue definido y discutido por diversos autores (Andrefsky 1994; Bamforth 1986; Bousman 1993; Kelly 1988; Koldehoff 1987; Nelson 1991; Carr 1994a, 1994 b; Odell 1996; Torrence 1989a, 1989b). De acuerdo con Binford, la organización tecnológica puede ser entendida “in terms of strategies thar are responsive to concrete situational variables" (Binford 1979:255). En términos generales, los instrumentos responden a las estrategias implementadas y son considerados como un medio para resolver problemas: "Tools are not ends in themselves but are used by people as part of a larger strategy for coping with their social and physical environment" (Torrence 1989b:58).

Los conceptos de conservado y expeditivo caracterizan a las dos estrategias tecnológicas más utilizadas para explicar el producto material que resulta de la implementación de las mismas. En general, fueron vinculados con sociedades de 


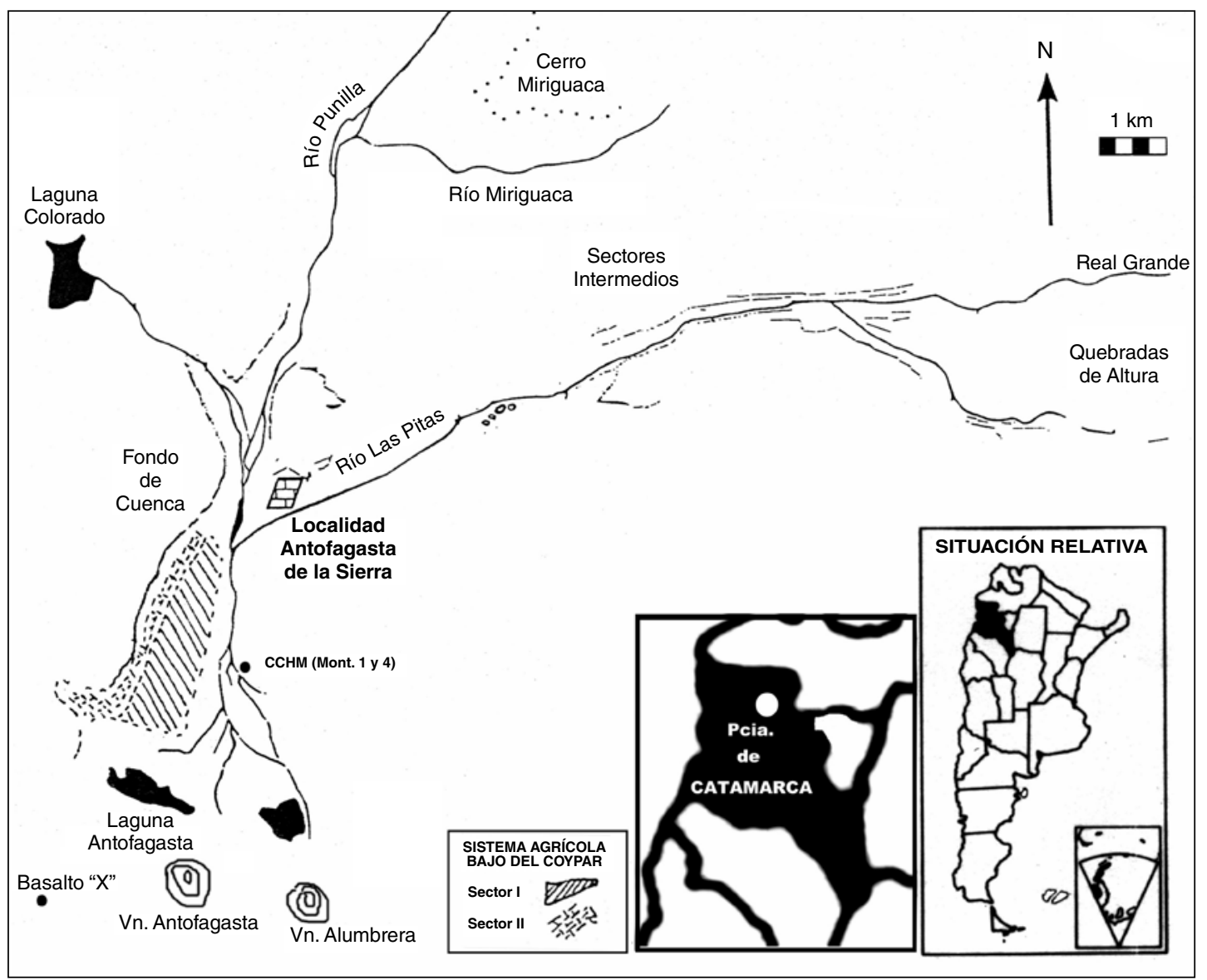

Figura 1. Área de estudio: ubicación del sitio Casa Chávez Montículos. Study area: Location of Casa Chávez Montículos site.

cazadores y recolectores, aunque algunos investigadores extendieron su aplicabilidad a sociedades con economías productoras (Escola 2000, 2004; Jeske 1992; Koldehoff 1987; Morrow 1987; Parry y Kelly 1987). En los últimos años, en nuestro país se reavivó la discusión sobre la operatividad de conceptos como el de organización tecnológica, conservación y expeditividad y su aplicabilidad al registro arqueológico (Bayón et al. 1995, 2001; Escola 2004).

El comportamiento relacionado con la selección de las estrategias implementadas fue atribuido a diversas variables y se basa en la evaluación de los costos involucrados en el uso eficiente del tiempo, energía y recursos, como por ejemplo: disponibilidad de materia prima, localización de las fuentes de aprovisionamiento, estrés temporal, manufactura, transporte y movilidad, variando el énfasis puesto en cada variable (Bamforth 1986; Binford 1979; Kelly 1992, 1999; Torrence 1989b; entre otros).

Siguiendo a Nelson, en este trabajo se considera que "Curation is a strategy of caring for tools and toolkits that can include advanced manufacture, transport, reshaping, and caching or storage" (Nelson 1991:62). De acuerdo con los resultados que se discutirán más adelante, se coincide con esta autora en cuanto a que la variable crítica que diferencia lo conservado de lo expeditivo es la preparación de los materiales en forma planificada antes de su uso (p.ej. adquisición y selección de la materia prima, manufactura de los artefactos y enmangue). De cualquier modo, no es conveniente ni productivo realizar una separación terminante entre las estrategias, y menos aún considerar a todo el contexto de un sitio bajo un solo tipo de estrategia. Es necesario tener en cuenta que ciertas opciones 
resultan mejores ante determinadas actividades. $\mathrm{Al}$ respecto, Nelson considera que:

\begin{abstract}
Expedient and curated plans can be interwoven, contributing to complexity of the eventual products in the archaeological record... It is crucial that curation and expediency not be perceived as mutually exclusive systems, but as planning options that suit different conditions within a set of adaptative strategies (Nelson 1991:65).
\end{abstract}

Además, esta misma autora señala que existe una tercera estrategia, que denomina comportamiento oportunístico, la cual contrastaría, según ella, con la expeditividad y que se trata de un comportamiento no planeado que responde a condiciones inmediatas, no anticipadas. Nelson, al referirse específicamente al producto material resultante de la implementación de las distintas estrategias, afirma que:

\section{Opportunistic designs and distributions are conditioned primarily by specific environmental and behavioral contexts, while designs from strategies of curation and expediency are conditioned by both the specific context and the broader context of planning... The various aspects of curation, expediency, and even opportunistic beha- vior have implications for the design and spatial distribution of tools and toolkits (Nelson 1991:65-66).}

Un aspecto importante en el estudio de las estrategias tecnológicas lo constituye el diseño de los artefactos y lo que encierra este concepto. En este caso, resulta más conveniente tratar las variables de diseño en forma conjunta con la discusión de los resultados, lo cual se presenta más adelante.

\section{Materiales y Métodos}

Se trabajó con dos colecciones, una experimental, conformada por 12 instrumentos replicados (sensu Nami 1992), y otra arqueológica, conformada por el total del material recuperado en las excavaciones de CCHM. Se trata de cuatro instrumentos enteros y 474 fragmentados, lo cual nos indica el alto índice de fragmentación de la muestra. Los subgrupos tipológicos clasificados incluyen: piezas enteras (ENT), fragmentos diferenciados (DIF) en los cuales es posible determinar a qué parte de la pieza entera corresponden, fragmentos no diferenciados con y sin filos (NDCF y NDSF), fragmentos indiferenciados (INDI) y preformas (PREF) (Figura 2) (Pérez 2006a, 2006b).

La materia prima utilizada en la manufactura de palas y/o azadas, en ambas colecciones, corresponde a rocas volcánicas que se clasifican entre basaltos y andesitas y se diferencian a nivel de cristalinidad y textura en las pastas. Los soportes se caracterizan por presentar formas tabulares o laminares, informalmente denominados 'lajas', de largos y anchos mucho mayores que el espesor. Otra característica es que la superficie de las discontinuidades es casi lisa y el tamaño del clasto corresponde a bloque (Pérez et al. 2006:221).

Aschero et al. (2002-2004), sobre la base de establecer una unificación en la denominación de materias primas líticas utilizadas para la talla de artefactos en la microrregión de Antofagasta de la Sierra, proponen denominar a dicha materia prima como vulcanita (Vc 8).

La metodología de la investigación se basó en tres vías de análisis: (a) el análisis técnico-morfológico y morfológico-funcional de los materiales bajo estudio,

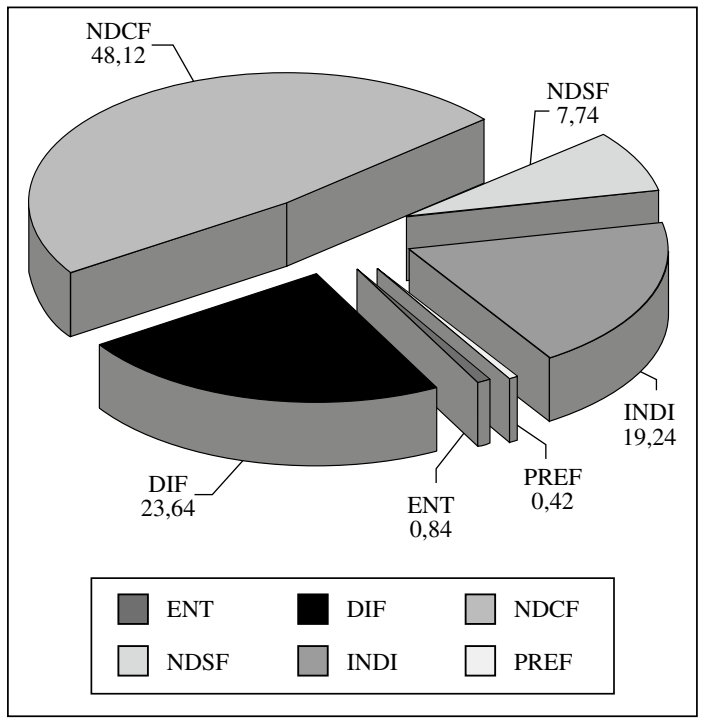

Figura 2. Casa Chávez Montículos (Montículos 1 y 4). Distribución porcentual de palas y/o azadas por Subgrupo Tipológico $(\mathrm{N}=478)$.

Casa Chávez Montículos site (Mounds 1 and 4). Distribution of shovels and/or hoes for Typological subgroup $(N=478)$. 
(b) la implementación de un Programa Experimental tendiente a la replicación de las diversas etapas de producción y uso de los artefactos y (c) el análisis de los rastros producidos por el uso en ambas colecciones: la arqueológica y la experimental de referencia. A continuación se reseñan brevemente las líneas de investigación (para una descripción detallada ver Pérez 1993, 2003a, 2003b, 2004, 2005, 2006a, 2006b y 2006-2007).

\section{Análisis técnico-morfológico y morfológico- funcional}

El análisis se efectuó siguiendo los lineamientos propuestos por Aschero $(1975,1983)$ y Pérez $(2003 a$, 2006b). Se siguieron cuatro pasos: (a) segmentación del conjunto, (b) orientación, ilustración y segmentación descriptiva de los instrumentos, (c) descripción técnico-morfológica y morfológico-funcional y (d) clasificación de subgrupos tipológicos.

Las piezas fueron divididas en dos secciones: cuerpo y pedúnculo. A su vez, se realizó la segmentación descriptiva (sensu Aschero 1983), para lo cual se han tomado en cuenta aspectos que pudieran dar un indicio certero de la ubicación del fragmento en relación a la pieza entera, tales como forma general del fragmento y borde, cambio en la curvatura del borde o evidencias macroscópicas de pulido y/o estrías. Los criterios utilizados para la clasificación diferencial de los subgrupos tipológicos se basaron en la segmentación descriptiva detallada anteriormente.

Es importante destacar que no se procedió a separar 'palas' y 'azadas' como grupos de instrumentos distintos a fin de no realizar una categorización a priori. En este sentido, todos los grupos tipológicos poseen una cuota de asignación funcional de los instrumentos para su clasificación y, en este caso en particular, es necesario profundizar en las investigaciones referidas a la experimentación de uso y la posterior determinación funcional de los instrumentos a través de análisis microscópicos de rastros de uso.

\section{Programa experimental}

El programa experimental estuvo dirigido a la realización de diversas actividades relacionadas con las diferentes etapas por las cuales pasaron los artefactos: (a) aprovisionamiento y selección de la materia prima, (b) replicación (sensu Nami 1992) de artefactos a fin de obtener una colección de referencia, (c) experimentación de enmangue y (d) experimentación de uso.

Los análisis efectuados en el conjunto artefactual de la colección arqueológica permitieron realizar una cuidadosa selección de la forma base y guiaron el trabajo de replicación. El trabajo de talla fue realizado con técnica de percusión directa. En la formatización y regularización de los bordes se aplicaron procedimientos de retalla y retoque marginal bifacial. Los instrumentos replicados fueron enmangados para su utilización, utilizando para ello técnicas de talabartería.

Teniendo en cuenta que las características del material de contacto, en este caso sedimento, inciden directamente en la formación de los rastros producidos por el uso en los instrumentos, se realizaron análisis granulométricos, ensayos para determinar el grado de compactación y de humedad, así como también ensayos químicos tendientes a conocer tanto la presencia/ausencia de materiales orgánicos, como los contenidos de componentes ácidos del suelo.

A fin de ajustar la metodología de experimentación, se realizaron ensayos piloto de uso con lajas sin manufactura previa y sin enmangar. $\mathrm{La}$ finalidad era observar si los filos naturales eran aptos para el laboreo de la tierra, si se producían rastros significativos en los mismos, y evaluar la cantidad y calidad del trabajo realizado con y sin enmangue.

La experimentación de uso se llevó a cabo en los campos de cultivo prehispánicos denominados Bajo del Coypar I-Sector 1, en las inmediaciones del sitio bajo estudio. Los instrumentos experimentales fueron utilizados en dos modos de acción (cavar y puntear) en forma separada. Las pruebas de uso se encararon con interrupciones de 500, 1.000 y 1.500 golpes o penetraciones en el sedimento, controlando diversas variables independientes (atributos del instrumento, atributos del material de contacto y acción del instrumento) como variables dependientes (estrías, pulidos, filos redondeados y microfracturas) (Richards 1988). Asimismo, se realizaron controles a fin de registrar las fracturas que pudieran ocurrir durante la producción y uso de los instrumentos en las actividades programadas. 


\section{Análisis de rastros de uso}

El análisis de los rastros producidos por el uso fue efectuado en 12 instrumentos replicados experimentalmente (Figura 3), y en cuatro instrumentos enteros (Figura 4) y 11 fragmentados (Figura 5) de la colección arqueológica. Las observaciones fueron realizadas mediante una lupa binocular (estéreo microscopio) Olimpus VMZ (BH2-PM-6), con magnificaciones entre 40x y 160x, y luz externa oblicua (con incidencia a $45^{\circ}$ respecto al plano de la pieza). Por lo tanto, los rastros observados se refieren sólo a esta escala de análisis.

El análisis fue dirigido a determinar el proceso de formación y las diferentes velocidades de aparición y desarrollo de los rastros. Se procedió a la comparación analógica de los rastros de utilización y las características formales entre el material arqueológico y el experimental. Finalmente, se realizó un estudio tendiente a establecer las relaciones existentes entre el comportamiento de uso y los rastros presentes en la colección experimental.

\section{Resultados}

\section{Análisis técnico-morfológico y morfológico- funcional}

A pesar de la variabilidad interna que presenta la muestra analizada, es posible adscribirla a un mismo estándar morfológico. De acuerdo con las características técnico-morfológicas, se trata de instrumentos formatizados por lascados de retalla y retoque marginal bifacial en todo su perímetro, manufacturados por percusión directa. La forma general del cuerpo es semielíptica con presencia de pedúnculo y, en algunos casos, la morfología del cuerpo aparece modificada como resultado de eventos de reactivación del filo apical. Asimismo, se ha observado que los bordes del pedúnculo se encuentran embotados intencionalmente con técnicas de retoque para la regularización de filos y bordes, lo cual se infiere, a partir de las experimentaciones llevadas a cabo, que es para facilitar la práctica de enmangamiento.
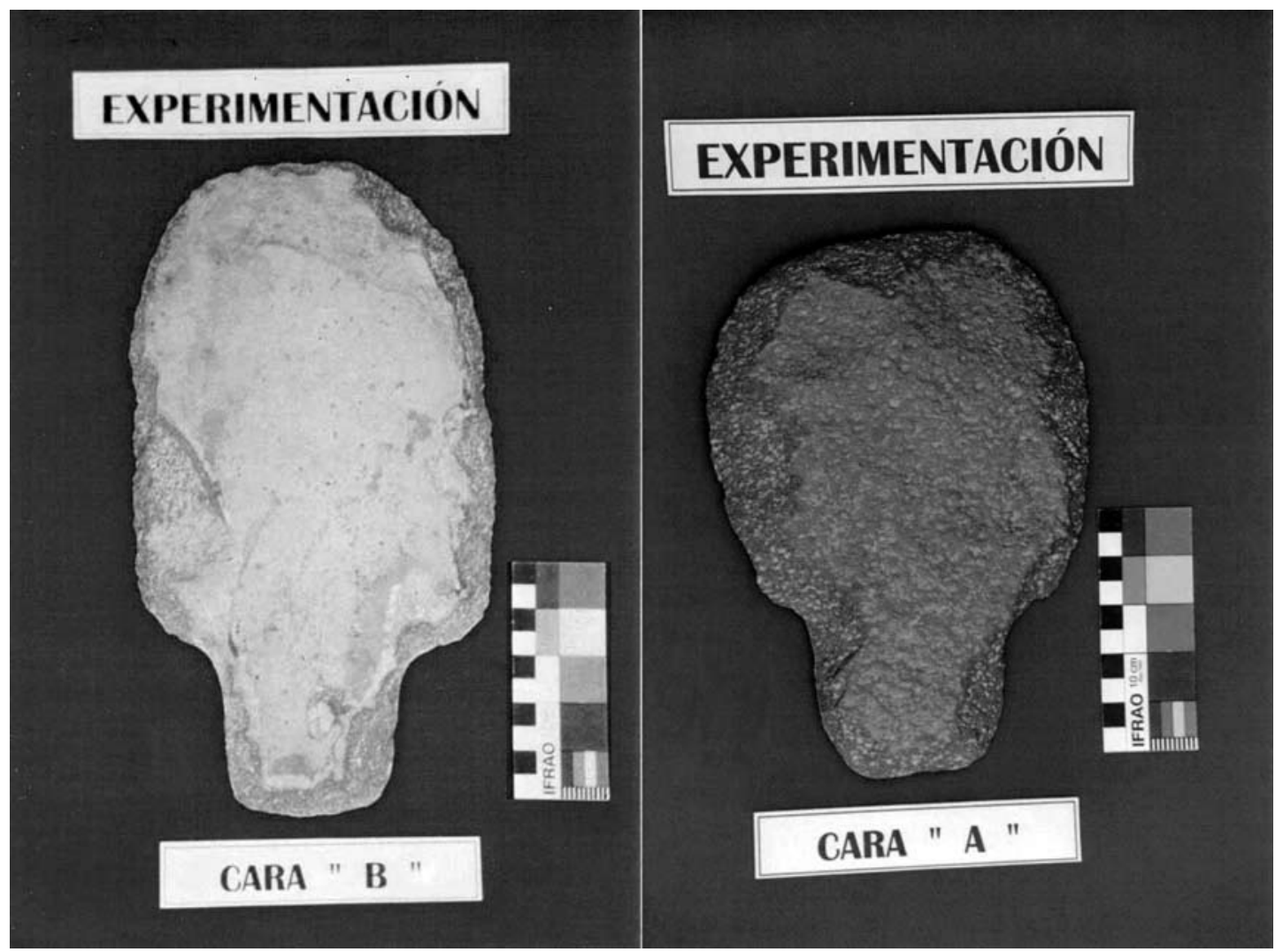

Figura 3. Colección experimental: instrumentos replicados.

Experimental collection: replicated instruments. 


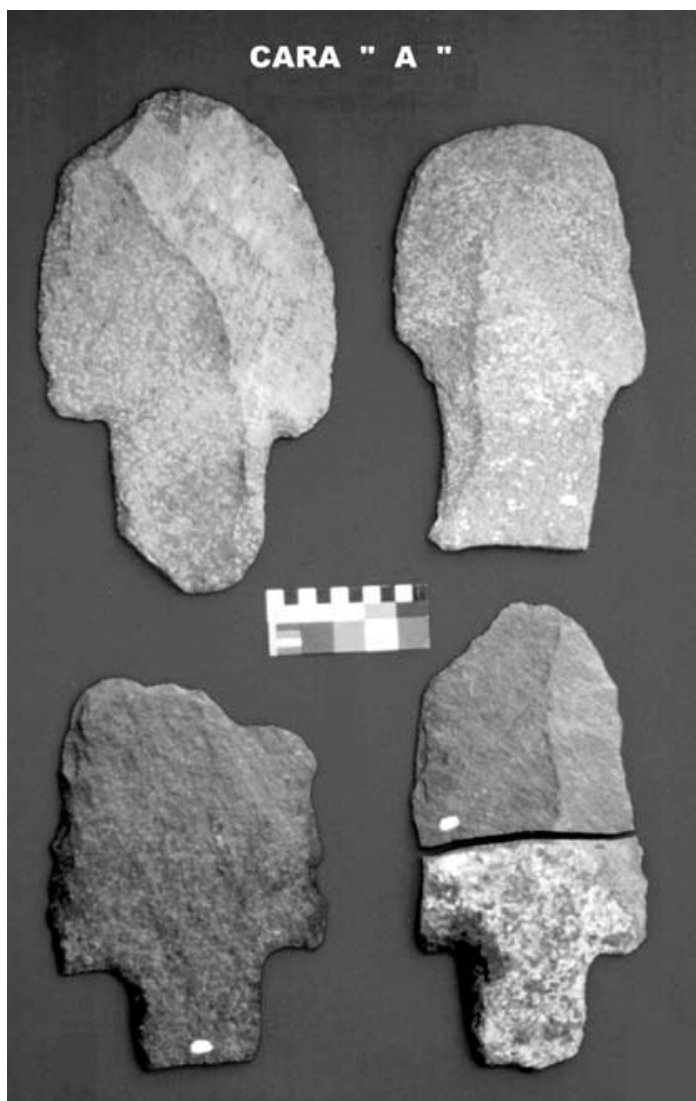

Figura 4. Casa Chávez Montículos (Montículos 1 y 4). Palas y/o azadas, instrumentos enteros.

Casa Chávez Montículos site (Mounds 1 and 4). Shovels and/ or hoes, whole instruments.

La media aritmética de las dimensiones absolutas de los instrumentos enteros es de 280,75 mm de longitud y de $168,75 \mathrm{~mm}$ de ancho. Existe una tendencia a registrarse espesores mayores en la porción correspondiente al pedúnculo e inflexión cuerpo-pedúnculo (12 y 13,27 mm, respectivamente), en relación con el cuerpo de las piezas $(7,75 \mathrm{y} 11,75 \mathrm{~mm}$ en el cuerpo apical y medio, respectivamente). Con respecto al peso de los instrumentos enteros, éste oscila entre 549 y $1.056 \mathrm{~g}$, siendo la media de $805,75 \mathrm{~g}$.

\section{Programa experimental}

La materia prima utilizada en la manufactura de la colección experimental fue $\mathrm{Vc} 8$ (sensu Aschero et al. 2002-2004), proveniente de la fuente de aprovisionamiento que se localiza sobre la margen izquierda de las coladas de los volcanes AntofagastaLa Alumbrera (Los Negros), distante entre 5 y $7 \mathrm{~km}$

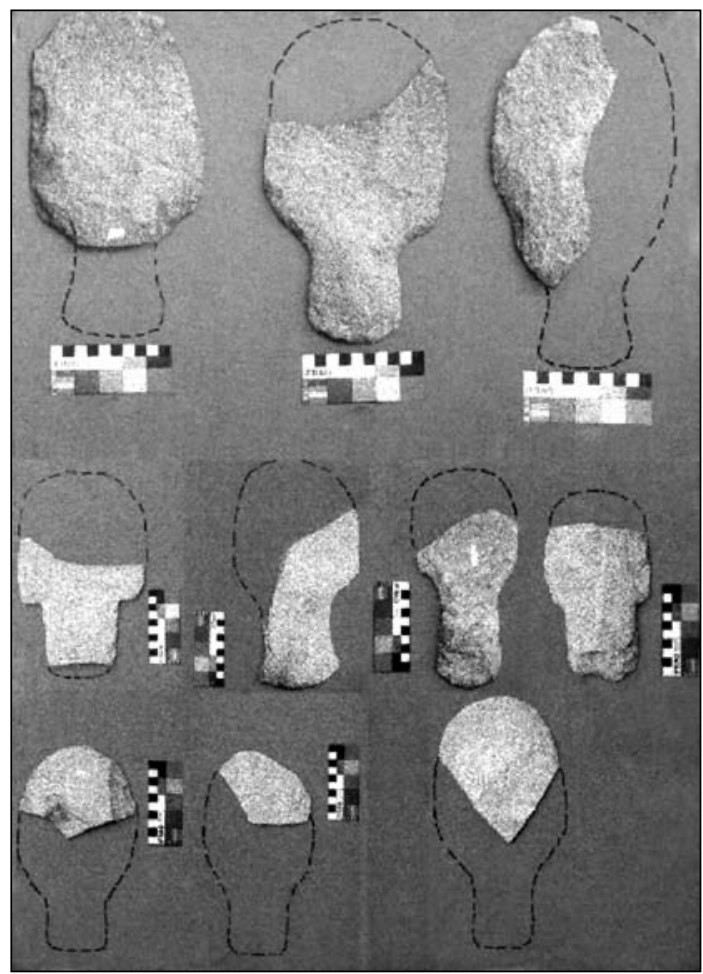

Figura 5. Casa Chávez Montículos (Montículos 1 y 4). Fragmentos diferenciados de palas y/o azadas.

Casa Chávez Montículos site (Mounds 1 and 4). Differentiated fragments of shovels and/or hoes.

del sitio CCHM (Escola 2000) y constituye lo que Nami (1992) denomina como "fuente de materia prima primaria". Como se advierte, la materia prima se localiza muy próxima al sitio, además es abundante y de fácil acceso.

Los soportes se presentan en la fuente de aprovisionamiento en diferentes tamaños y grosores, razón por la cual fue necesaria una cuidadosa selección de la forma base para la manufactura de los artefactos. Las dimensiones absolutas de los clastos recogidos para los trabajos de replicación no superan un largo de $550 \mathrm{~mm}$, un ancho de $355 \mathrm{~mm}$ y un espesor de $55 \mathrm{~mm}$, siendo su peso aproximado de $2.000 \mathrm{~g} \mathrm{o}$ más por unidad, en algunos casos puede duplicar este peso.

Con respecto al material de contacto, en términos generales, se puede decir que se trata de un sedimento muy friable, arenoso, con clastos de granulometría variada y prácticamente nula la presencia de arcilla. En cuanto a los componentes orgánicos y ácidos, en todas las muestras se registra un porcentaje muy bajo de los mismos. 
El uso experimental de palas y/o azadas en diferentes actividades relacionadas con el laboreo de la tierra permitió aproximarnos a su funcionalidad. Las actividades de roturado del terreno y de formación de un bordo, con los modos de acción puntear y cavar, respectivamente, se lograron de manera satisfactoria. La forma general de los instrumentos y los ángulos de los filos activos resultaron aptos para las tareas de laboreo de la tierra. Además, la utilización de los instrumentos con y sin enmangue permitió determinar que para obtener una mayor eficacia en las tareas, las palas y/o azadas debieron utilizarse enmangadas. Esto fue comprobado a partir del control de variables como: superficie trabajada, tiempo, energía y eficiencia para realizar las actividades, permitiendo establecer una mayor eficacia, practicidad y rapidez para realizar las actividades. Efectivamente, con la misma cantidad de golpes o penetraciones en el sedimento, la superficie trabajada fue mayor con los instrumentos enmangados. Por otro lado, si bien en el proceso de enmangue de los instrumentos o en un eventual recambio del mismo es necesario un aporte especial de tiempo y precisión para el posterior uso en las actividades programadas, el tiempo y el esfuerzo involucrado en las actividades de laboreo son mayores con las piezas sin enmangar.

\section{Análisis de rastros de uso}

La comparación analógica de los rastros de utilización y las características formales entre el material arqueológico y el experimental permitió establecer la localización, distribución y orientación de los mismos, así como también el grado de desarrollo que presentan:

Estrías: en la colección experimental tanto en los segmentos apicales como en los laterales del cuerpo, laterales del pedúnculo e inflexión cuerpopedúnculo, hay un predominio de estrías oblicuas $(50 \%)$, le siguen las perpendiculares $(28 \%)$ y las horizontales (22\%). En cambio, en la evidencia arqueológica, en la porción apical la representatividad de oblicuas y perpendiculares es compartida $(42 \%)$ y las horizontales $(17 \%)$ tienen una muy distanciada representatividad en relación a las otras dos orientaciones, mientras que en los segmentos correspondientes a los laterales del cuerpo, laterales del pedúnculo e inflexión cuerpo-pedúnculo, la tendencia es similar a la generalidad observada en la colección experimental. Esta tendencia se observa por igual en ambas caras de las piezas y en ambos modos de acción implementados. Con respecto a las características de las estrías, son homogéneas en todos los ejemplares estudiados.

Con respecto a las estrías observadas en la porción correspondiente al pedúnculo, estas son diagnósticas para la determinación del proceso de enmangue cuando comparamos los datos obtenidos en las pruebas piloto, donde no se practicó enmangue alguno, y el uso de los instrumentos con enmangue. En el primer caso, no se perciben rastros semejantes a los que se observan en el material arqueológico, en cambio, en el segundo caso los rastros producidos por el enmangue guardan similitud con los observados en el registro arqueológico. Se observa una tendencia a registrarse una mayor preponderancia de estrías con orientación oblicua, en detrimento de las perpendiculares y horizontales (Figura 6). Es importante destacar que el tiempo de uso de los instrumentos y la cantidad de episodios de enmangue que se efectúan en cada pieza es crucial para la formación de los rastros con la intensidad que se observan en la evidencia arqueológica.

Pulidos: en la muestra arqueológica analizada se observa pulido en algún grado de desarrollo y éste se manifiesta en el filo apical y en el cuerpo

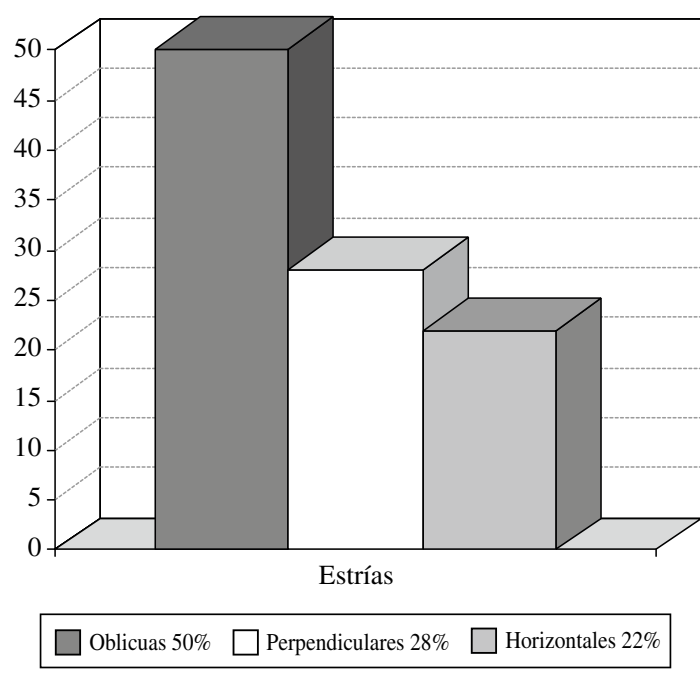

Figura 6. Colección experimental. Distribución porcentual de estrías en el pedúnculo, según su orientación respecto del filo apical $(\mathrm{N}=12)$.

Experimental collection. Distribution of grooves in the stem, according to their orientation with respect the tip $(N=12)$. 
de las piezas en su porción próxima al filo apical. Predominan, 'muy brillantes' y 'brillantes'. En la colección experimental el pulido es homogéneo en cuanto a sus características y se presenta 'mate'. Lo interesante fue detectar que en los instrumentos utilizados en el modo de acción 'cavar' el pulido se observa en la porción apical, mientras que en 'puntear', el pulido también se extiende hacia los bordes laterales del cuerpo en su parte próxima al filo apical.

Filos redondeados: en ambas colecciones la presencia de filos redondeados se constató en los segmentos apicales y laterales del cuerpo en su parte próxima al filo apical. En la colección experimental la presencia de filos redondeados es homogénea; en cuanto a sus características, en todas las piezas se presentan 'levemente redondeados'. En cambio, en la muestra arqueológica están 'fuertemente redondeados' en la mayoría de las piezas; los que se observan 'levemente redondeados' se encuentran en algunos casos en un grado mayor de desarrollo que los observados en la colección experimental.

Microfracturas: en la evidencia arqueológica, las microfracturas fueron observadas en los segmentos correspondientes al filo apical y laterales del cuerpo, mientras que en la colección experimental se observaron sólo en la porción apical de algunos de los ejemplares.

De acuerdo con la presencia, cantidad y grado de desarrollo de rastros observados en este nivel de análisis, se puede afirmar que es necesario un tiempo prolongado de uso de los instrumentos para que adquieran las características e intensidad que se observan en la evidencia arqueológica como, por ejemplo, para que el pulido se presente con el brillo intenso característico del material de CCHM.

La presencia de rastros de microdesgaste indica si un instrumento fue utilizado y cuál fue el área específica involucrada en el proceso de uso. Además, la distribución del pulido o la orientación de las líneas de fractura y estriaciones también indican el movimiento de un instrumento (longitudinal o transversal al filo, o de rotación), es decir que el movimiento de uso es inferido a través de la combinación de variables tales como la morfología del filo, la distribución del pulido, el desgaste de un filo y las microfracturas o estriaciones presentes.

El estudio de las relaciones existentes entre el comportamiento de uso y los rastros producidos por el uso, tales como estrías, pulidos, filos redondeados y microfracturas, permitió determinar el área usada del instrumento, la acción del instrumento y la actividad desarrollada. El área usada del instrumento corresponde al segmento apical y laterales del cuerpo de las piezas, en su porción próxima al filo apical. Para determinar la acción del instrumento, los rastros que podrían dar algún indicio de la forma concreta de utilización son la presencia de pulidos y filos redondeados, no solamente en el filo apical utilizado, como ocurre con el modo 'cavar', sino que se manifieste también en el área superior de los laterales del cuerpo de las piezas, como se observó en el modo 'puntear'. Con respecto a las estrías, en ambos modos de acción implementados en la experimentación de uso, la cantidad, ubicación y características resultaron ser similares.

La actividad desarrollada estuvo vinculada con tareas de laboreo de la tierra, implicando una amplia gama de tareas que son posibles de realizar. Éstas incluyen tanto las relacionadas con la agricultura como las tendientes a responder a las necesidades de un campamento base, tales como limpieza de basurales y lugares de habitación, excavación de pozos para almacenamiento de diversos productos, etc. Esto implica un empleo de los instrumentos con diferentes modos de acción, que son necesarios para llevar adelante las diferentes tareas antes mencionadas.

\section{Discusión}

A fin de discutir los resultados obtenidos, considero necesario abordar la utilización de conceptos referidos al diseño de los artefactos. Las variables referidas a diseño fueron tratadas por diversos autores. Bleed, en su estudio de las estrategias entre cazadores, habla de diseños alternativos para optimizar la disponibilidad de cualquier sistema técnico: "reliability" y "maintainability" (Bleed (1986), los cuales tienen diferentes aplicaciones óptimas y diferentes características físicas. En el primero los instrumentos son hechos para cuando se los necesita, mientras que en el segundo pueden ser hechos fácilmente para cumplir con la función ante una eventual fractura o si no son apropiados para realizar las tareas a mano.

El diseño se refiere a variables conceptuales de utilidad que condicionan las formas de los instrumentos y la composición de los equipos (toolkit) 
(Nelson 1991). En esta discusión se partió del modelo propuesto por Nelson, quien toma en cuenta cinco variables para hablar de diseño: confiabilidad, mantenibilidad, transportabilidad, flexibilidad y versatilidad (Nelson 1991).

Un elemento básico en un diseño confiable es el sobrediseño (Bleed 1986), especialmente de determinadas partes del artefacto, que contarán con refuerzos que posibiliten su enmangue, como es el caso de la morfología del pedúnculo que se observa en palas y/o azadas. Lo que le otorga confiabilidad son justamente esas partes sobrediseñadas y fortalecidas, no solamente para facilitar el enmangue sino que también para otorgarle al instrumento un refuerzo extra ante posibles fracturas producidas por la fuerza ejercida al realizar las actividades (más adelante retomaré este tema).

Un aspecto importante a tener en cuenta es que el reemplazo del instrumento ante una eventual fractura debe ser simple y estandarizado, y debe ocurrir con anticipación al uso para no interferir con el tiempo de trabajo (Nelson 1991). En este sentido, y teniendo en cuenta la abundante localización de palas y/o azadas en el campamento base, en relación con los sectores de cultivo, coincidimos con Keeley (1982) en cuanto a que los instrumentos tienden a acumularse en contextos arqueológicos cuando y donde son reemplazados en sus mangos, lo cual no necesariamente ocurre cuando y donde son usados por última vez. Con respecto a los instrumentos enteros, en CCHM se registró una concentración de varios de ellos junto a un fogón. Además, la gran cantidad de desechos y fragmentos presentes en el campamento base indicarían que este tipo de material se halla en el sitio habitacional como resultado de la manufactura y también de la reactivación de los instrumentos, a fin de obtener los mismos en forma anticipada al uso específico en las actividades programadas.

Otra característica de un sistema confiable es que los instrumentos son manufacturados por especialistas (Bleed 1986). De acuerdo con la replicación de artefactos llevada a cabo en el programa experimental, se pudo comprobar que, si bien no es necesario un tiempo prolongado de manufactura para obtener artefactos con las características de los que nos ocupan aquí, se requiere un cierto grado de conocimiento y manejo de la técnica de talla para lograr el producto final, es decir, un adecuado conocimiento tecnológico para lograr el producto final sin que se produzcan fracturas en el proceso de manufactura, especialmente en la etapa de confección del pedúnculo.

Las palas y/o azadas responden a un diseño confiable. Evidencia de ello es la presencia de reactivación de los filos apicales, el costo en lo referente a la manufactura del pedúnculo y en el transporte, ya que a pesar de que la fuente de aprovisionamiento está próxima y es de fácil acceso, el transporte de los soportes al campamento base para efectuar la manufactura es bastante difícil si tenemos presente el tamaño de los mismos, tal como se mencionó anteriormente.

A pesar de que se esperaría para un diseño confiable la condición de portable, es necesario aclarar que esta condición de portabilidad de los instrumentos fue discutida por los arqueólogos en relación a la alta movilidad de grupos cazadores. En nuestro caso, las palas y/o azadas, de contextos sedentarios agropastoriles, no cumplen esta condición de portabilidad, ya que poseen grandes dimensiones y su peso, aproximado en $1.000 \mathrm{~g}$ por unidad, no las hacen muy apropiadas para el traslado continuo. No obstante ello, teniendo en cuenta el costo en la adquisición de formas bases aptas para la manufactura y la especialización en cuanto a la técnica necesaria para obtener el producto final, el costo invertido en la dificultad de portabilidad de los instrumentos se compensa con la seguridad y confiabilidad de contar con un reemplazo estandarizado y adecuado en caso de ser necesario para proseguir con las tareas.

Un diseño mantenible es aquel que puede ser hecho o modificado para multipropósitos (Bleed 1986). Se trata de un diseño flexible en los términos de Nelson (1991), quien también distingue los instrumentos que mantienen una forma generalizada, es decir, que no cambian la forma para satisfacer una variedad de necesidades, en este último caso los denomina diseños versátiles. La mantenibilidad, para Nelson, se obtiene en el costo de tiempo invertido en la manufactura y uso. En el caso específico de palas y/o azadas líticas, las consideraciones de flexible y versátil antes vertidas no se cumplirían. En principio, si bien se pudo comprobar experimentalmente la eficiencia de este tipo de instrumentos para realizar tareas de laboreo de la tierra, es necesario proseguir con las investigaciones encaradas en el programa experimental a fin de comprobar si son aptos para otro tipo de tareas fuera de las relacionadas con el laboreo de la tierra o, en términos de Nelson, para satisfacer una variedad de necesidades. 
Por otro lado, siguiendo a Nelson:

\begin{abstract}
All design flexibility requires changing form through reworking and replacement as part of the use of the toolkit. This implies the incorporation of simple repair kits in flexibly designed toolkits (Bleed 1986). In prehistoric technologies these might include bone and stone hammers, prepared cores, and resin, among other items (Nelson 1991:71-72).
\end{abstract}

En el caso concreto de las palas y/o azadas, en el toolkit es imprescindible que se incluyan, además de instrumentos de repuesto por una eventual fractura, tientos para efectuar el recambio de instrumentos en el soporte, es decir, en el mango de madera.

Con respecto a la transportabilidad, se espera que los artefactos y equipos (toolkit) sean pequeños y livianos para su fácil traslado. Como se mencionó anteriormente, esta condición no se cumple en nuestro caso, pero es necesario tener en cuenta que para satisfacer eficientemente con la función para la cual fueron creados, no servirían instrumentos de pequeño tamaño, ya que las actividades requieren artefactos con las dimensiones y morfología como las que ostentan las palas y/o azadas. En este sentido, Nelson considera que:

... caution must be exercised in considering tool size as an index of portability. Tool size (not toolkit size) should not be evaluated without consideration of the extent of reduction of each tool, the materials from which tools were made, the tasks for which they were intended and used, and their organizational role in the technology (Nelson 1991:76).

Además, según Nelson, los instrumentos transportados deben ser utilizados intensivamente antes del reemplazo o descarte. En el caso que nos ocupa, el análisis efectuado en el registro arqueológico revela que la reactivación presente en los filos utilizados fue sobre superficies altamente pulidas y desgastadas por el uso.

El mantenimiento de palas y/o azadas, entendido como la búsqueda de una larga vida útil, además de estar evidenciado en la reactivación de los filos utilizados, está relacionado con el costo del aprovisionamiento de la materia prima, del transporte y de la manufactura, y se relaciona con la preparación planificada, anticipada al uso efectivo de los instrumentos. No se vincula con una estrategia orientada a la preservación de la materia prima en los términos planteados por Bamforth (1986), sino con la cuidadosa y eficiente recolección de formas bases aptas para la manufactura de los instrumentos, y con la búsqueda de prolongar la vida útil de los artefactos, dado el trabajo invertido para su producción. Tampoco se trata de prácticas relacionadas con estrategias de movilidad originadas por la distancia a la fuente de aprovisionamiento de la materia prima ya que, como se mencionó anteriormente, la misma se localiza muy próxima al sitio, es abundante y de fácil acceso. Es un recurso disponible, aunque de no muy fácil transportabilidad, dado el gran tamaño de las formas bases. Teniendo en cuenta lo expresado, considero que se trata de comportamientos tecnológicos específicos que hacen operativamente viable la conservación de los instrumentos.

Por último, hay que destacar que determinadas variables de diseño fueron formuladas y pensadas para el comportamiento de grupos cazadores y por consiguiente en los equipos instrumentales asociados a ellos. Es necesario extender este tipo de estudios a contextos de grupos sedentarios con economías productoras de alimentos.

En otro orden de cosas, la práctica de enmangue es vinculada habitualmente con la preparación del instrumento en forma anticipada a su uso y es considerada como indicativa de instrumentos conservados o, al menos, que ocurra con más probabilidad en estrategias conservadas. Keeley (1982:799) afirma que un instrumento es enmangado por varias razones: para aumentar la fuerza que pueda ser ejercida durante el trabajo, para reforzar la eficacia o precisión, para permitir la formación de bordes cortantes y para conservar o disminuir la posibilidad de pérdida mediante el aumento del tamaño de los utensilios completos. Algunas de estas razones tienen un argumento funcional, ya que son prácticas orientadas al uso eficiente de los instrumentos, entendidas como la mejor opción para realizar las tareas de una manera más eficiente. Se trata de una optimización en la selección de estrategias tecnológicas orientadas a la conservación de los instrumentos. En el caso de las palas y/o azadas considero que el enmangue podría deberse a la búsqueda funcional del instrumento, es decir, aumentar la fuerza ejercida y reforzar la eficacia en la labor llevada a cabo. 
Keeley (1982), además, agrega que es probable que los utensilios enmangados tengan rasgos especiales que se relacionen con sus acondicionamientos de enmangamiento, tales como pedúnculos, escotaduras bilaterales o basales, hombros y bordes proximales embotados. En este sentido, tal como se mencionó anteriormente, los instrumentos en estudio presentan rasgos morfológicos que los hacen aptos para enmangar.

Los diseños confiables también poseen encastres seguros (Nelson 1991), de alta calidad y con partes reforzadas que le otorgan resistencia (Bleed 1986), lo cual fue comprobado en esta investigación a partir de los resultados de los análisis técnico-morfológicos y con la implementación del programa experimental.

Las investigaciones llevadas a cabo permiten afirmar que las palas y/o azadas líticas de CCHM fueron enmangadas para su utilización. Los resultados de los análisis técnico-morfológicos efectuados revelan que su morfología hace posible el enmangue, de otro modo la laja en estado natural, es decir, sin manufactura, no permite realizar su enmangue de una manera firme debido a las dimensiones de la misma. Además, el cuidadoso tratamiento en la confección de estos instrumentos, para lo cual es necesario un adecuado conocimiento por parte del operador durante la técnica de talla, especialmente en la porción correspondiente al pedúnculo, no se justificaría si los instrumentos fueran a ser utilizados sin enmangue. Por último, de acuerdo con los resultados obtenidos, las estriaciones observadas en la porción correspondiente al pedúnculo son diagnósticas para la determinación del proceso de enmangue.

\section{Conclusiones}

Esta presentación pretendió aportar información para acercarnos al estudio de la organización tecnológica a través de datos empíricos. Las evidencias presentadas en cuanto a las estrategias puestas en práctica para la adquisición de la materia prima, la manufactura, el uso y el mantenimiento de las palas y/o azadas líticas constituyen indicadores que sustentan la hipótesis de la implementación de estrategias tecnológicas dirigidas a la conservación de los artefactos.

Según Bousman (1993), el objetivo de un diseño es lograr una mayor eficiencia en cuanto al costo-beneficio de los instrumentos, y la eficiencia puede ser medida por lo menos en cuatro criterios: menor tiempo de producción, mayor vida útil, mayor eficiencia y mayor volumen de producción.

El diseño de las palas y/o azadas permite sostener que se trata de instrumentos estandarizados, enmangados para su utilización y con una larga vida útil, lo cual implicaría un costo medido en inversión de tiempo y energía dedicada a la obtención del producto final y un costo de tiempo, trabajo y efectividad para realizar las actividades, lo cual queda evidenciado en la importancia de una buena selección de la forma base para obtener un buen producto de talla, significando un costo en la obtención de la materia prima. Aunque la proximidad y accesibilidad a la fuente de materia prima permite el aprovisionamiento de los soportes necesarios, existe un costo referido a las dificultades del transporte de los mismos, dado el gran tamaño y peso detallados anteriormente.

En cuanto a la manufactura, las palas y/o azadas no tienen formatización sumaria; por el contrario, fueron confeccionadas por retalla y retoque, con regularización de bordes y embotamiento intencional de determinados filos de la pieza. La formatización de este tipo de instrumentos requiere el conocimiento de una técnica especializada para lograr el producto final, la cual insume una importante inversión de trabajo para su producción.

El enmangue se realiza generalmente en instrumentos conservados y, en el caso que nos ocupa, fue posible demostrar el enmangamiento de los instrumentos, el cual implica un costo medido en tiempo, trabajo y eficiencia.

La presencia y grado de desarrollo de los rastros de uso indican la utilización de los instrumentos por un tiempo prolongado, lo que permite hablar de una larga vida útil.

En síntesis, las evidencias indican que las palas y/o azadas líticas aquí analizadas son el resultado material de comportamientos tendientes a la conservación de los instrumentos y que los mismos respondían a un plan de anticipación al uso efectivo en las actividades programadas.

Agradecimientos: A Víctor por su ayuda en el procesamiento de las imágenes. A Paola Ramundo por la corrección del abstract. Al INAPL por brindarme el lugar de trabajo. A la Pontificia Universidad Católica Argentina por la asistencia financiera. A los evaluadores de este trabajo por sus comentarios, los cuales enriquecieron el mismo. 


\section{Referencias Citadas}

Andrefsky, W.Jr.

1994 Raw-material availability and the organization of technology. American Antiquity 59:1:21-34.

Aschero, C.A.

1975 Ensayo para una clasificación morfológica de artefactos líticos aplicada a estudios tipológicos comparativos. Informe a CONICET. Buenos Aires. Manuscrito en posesión del autor.

1983 Ensayo para una clasificación morfológica de artefactos líticos. Apéndice A y B. Cátedra de Ergología y Tecnología. Universidad de Buenos Aires. Manuscrito en posesión del autor.

Aschero, C.A., P.S. Escola, S. Hocsman y J. Martínez 2002-2004 Recursos líticos en la escala microregional Antofagasta de la Sierra, 1983-2001. Arqueología 12:9-36.

Bamforth, D.B.

1986 Technological efficiency and tool curation. American Antiquity 51:1:38-50.

Bayon, C., P.S. Escola y N. Flegenheimer

1995 Organización tecnológica: usos y abusos de esta perspectiva. Arqueología 5:179-186.

$2001 \mathrm{La}$ expeditividad y su registro arqueológico: un tema olvidado. Arqueología 11:261-270.

Binford, L.R.

1969 Stone tools and human behavior. Scientific American 220:4:70-84.

1973 Interassemblage variability-the Mousterian and the "functional" argument. En The Explanation of Culture Change: Models in Prehistory, editado por C. Renfrew, pp. 227-254. Duckworth Press, London.

1979 Organization and formation processes: Looking at curated technologies. Journal of Anthropological Research 35:3:255-273.

1980 Willow smoke and dogs' tails: hunter-gatherer settlement systems and archaeological site formation. American Antiquity 45:1:4-20.

Bleed, P.

1986 The optimal design of hunting weapons: maintainability or reliability. American Antiquity 51:4:737-747.

Bousman, C.B.

1993 Hunter-gatherer adaptations, economic risk and tool design. Lithic Technology 18(1-2):59-86.

Carr, P.J.

1994a Technological organization and prehistoric huntergatherer mobility: examination of the Hayes site. En The Organization of North American Prehistoric Chipped Stone Tool Technologies, editado por P.J. Carr, International Monographs in Prehistory. Archaeological Series 7, pp. 35-44. Ann Arbor, Michigan.

1994b The organization of technology: impact and potential. En The Organization of North American Prehistoric Chipped Stone Tool Technologies, editado por P.J. Carr, International Monographs in Prehistory, Archaeological Series 7, pp. 1-8. Ann Arbor, Michigan.

Escola, P.S.

2000 Tecnología Lítica en Sociedades Agro-Pastoriles Tempranas. Tesis Doctoral. Facultad de Filosofía y Letras, Universidad de Buenos Aires, Buenos Aires.
2004 La expeditividad y el registro arqueológico. Actas XV Congreso de Arqueología Chilena. Chungara Revista de Antropología Chilena Tomo 1:49-60.

Jeske, R.J.

1992 Energetic efficiency and lithic technology: an upper Mississippian example. American Antiquity 57:3:467481.

Keeley, L.H.

1982 Hafting and retooling: effects on the archaeological record. American Antiquity 47:4:798-809.

Kelly, R.L.

1988 The three sides of a biface. American Antiquity 53:4:717734.

1992 Mobitity/sedentism: concepts, archaeological measures, and effects. Annual Reviews of Anthropology 21:43-66.

1999 Theoretical and archaeological insights into foraging strategies among the prehistoric inhabitants of the stillwater march wetlands. En Understanding Prehistoric Lifeways in the Great Basin Wetlands: Bioarchaeological Reconstruction and Interpretation, editado por B. Hemphill and C.S. Larsen. University of Utah Press, Salt Lake City, en prensa. Manuscrito en poder del autor, facilitado durante el curso dictado en 1999, Buenos Aires.

Koldehoff, B.

1987 The Cahokia flake tool industry: socioeconomic implications for late prehistory in the central Mississippi valley. En The Organization of Core Technology, editado por J.K. Johnson and C.A. Morrow, pp. 151-185. Westview Press, Boulder and London.

Nami, H.G.

1992 El Subsistema tecnológico de la confección de instrumentos líticos y la explotación de los recursos del ambiente: Una nueva vía de aproximación. Actas X Congreso Nacional de Arqueología Argentina. Shincal Tomo 2:33-53.

Nelson, M.

1991 The study of technological organization. En Archaeological Method and Theory, editado por M.B. Schiffer, Vol. 3, pp. 57-100. University of Arizona Press, Tucson.

Morrow, C.A.

1987 Blades and Cobden Chert: A technological argument for their role as markers of regional identification during the Hopewell period in Illinois. En The Organization of Core Technology, editado por J.K. Johnson and C.A. Morrow, pp. 119-149. Westview Press, Boulder and London.

Odell, G.H.

1996 Economizing behavior and the concept of "curation". En Stone Tools. Theoretical Insights into Human Prehistory, editado por G.H. Odell, pp. 51-80. University of Tulsa, Oklahoma, Plenum Press, New York and London.

Olivera, D.E.

1991a El Formativo en Antofagasta de la Sierra (Puna Meridional Argentina): análisis de sus posibles relaciones con contextos arqueológicos Agro-alfareros Tempranos del NOA y Norte de Chile. Actas XI Congreso Nacional de Arqueología Chilena (1988), Tomo II:61-78. Museo Nacional de Historia Natural, Sociedad Chilena de Arqueología, Santiago.

1991b Tecnología y Estrategias de Adaptación en el Formativo (Agro-Alfarero Temprano) de la Puna Meridional Argentina. Un Caso de Estudio: Antofagasta de la Sierra (Pcia. de 
Catamarca, R.A.). Tesis Doctoral en Ciencias Naturales, Facultad de Ciencias Naturales, Universidad Nacional de La Plata, La Plata.

Olivera, D.E. y A. Nasti

1993 Site formation processes in the Argentine Northwest Puna: Taphonomic researches on archaeofaunistic record preservation. Arqueología Contemporánea 4:85-98.

Olivera, D.E. y S. Vigliani

2000-2002 Proceso cultural, uso del espacio y producción agrícola en la Puna meridional argentina. Cuadernos del Instituto Nacional de Antropología y Pensamiento Latinoamericano 19:459- 481.

Parry, W.J. y R.L. Kelly

1987 Expedient core technology and sedentism. En The Organization of Core Technology, editado por J.K. Johnson and C.A. Morrow, pp. 285-304. Westview Press, Boulder and London.

Pérez, $\mathrm{S}$.

1993 Informe de los primeros ensayos experimentales sobre azadas y/o palas líticas (Antofagasta de la Sierra/Catamarca). Palimpsesto. Revista de Arqueología 3:139-149.

2003a Experimentación y Análisis de Microdesgaste de "Palas y/o Azadas" Líticas de Antofagasta de La Sierra (Catamarca). Tesis de Licenciatura, Facultad de Filosofía y Letras, Universidad de Buenos Aires.

2003b Aproximación experimental aplicada a la determinación funcional de palas y/o azadas líticas. Hombre y Desierto. Una Perspectiva Cultural 11:85-113.

2004 Experimentación de uso con palas y/o azadas líticas. Intersecciones en Antropología 5:105-117.

2005 Análisis de microdesgaste por uso de palas y/o azadas líticas de Antofagasta de la Sierra (Pcia. de Catamarca).
Aportes para su interpretación funcional. Hombre y Desierto. Una Perspectiva Cultural 12:23-46.

2006a Arqueometría de palas y/o azadas líticas aplicada a estudios experimentales. El caso de Antofagasta de la Sierra (Catamarca). Actas Primer Congreso Argentino de Arqueometría. Metodologías Científicas Aplicadas al Estudio de los Bienes Culturales, pp. 230-240. Editado por A. Pifferetti y R.E. Bolmaro, Rosario.

2006b Aportes metodológicos para el análisis de palas y/o azadas líticas. Mundo de Antes 5, en prensa.

2006-2007 Experimentación de enmangue de palas y/o azadas líticas. Boletín de Arqueología Experimental 7:73-85. Edición on-line. Servicio de Publicaciones de la Universidad Autónoma de Madrid (España), http://www. uam.es/departamentos/filoyletras/prearq/baex7.pdf

Pérez, S., P. Tchilingirian y P.S. Escola

2006 Caracterización de la materia prima utilizada en palas y/o azadas líticas de la Puna Argentina. Actas $1^{\text {er }}$ Congreso Argentino de Arqueometría. Metodologías Científicas Aplicadas al Estudio de los Bienes Culturales, pp. 218-229. Editado por A. Pifferetti y R.E. Bolmaro, Rosario.

Richards, T.H.

1988 Microwear Patterns on Experimental Basalt Tools. BAR International Series 460.

Torrence, $\mathrm{R}$.

1989a Tools as optimal solutions. En Time, Energy and Stone Tools, editado por R. Torrence, pp. 1-6. Cambridge University Press, Cambridge.

1989b Re-tooling: towards a behavioral theory of stone tools. En Time, Energy and Stone Tools, editado por R. Torrence, pp. 57-66. Cambridge University Press, Cambridge. 